\title{
Aportaciones de la folletería mexicana a la estadística decimonónica (1821-1910)
}

\author{
Walter Brem \\ THE BANCROFT LIJBRARY \\ UNIVERSITY OF CALIFORNIA, BERKILLYY
}

\begin{abstract}
Análisis cronológico del material estadístico incluido en los folletos recopilados en la base de datos del Instituto Mora
\end{abstract}

\section{INTRODUCCIÓN}

$\mathbf{S}$ e me pidió que realizara una presentación sobre la base de datos de la folletería en el campo de la estadística y durante el periodo de 1821 a 1910 ya que es un tema que está presente a lo largo de esa época. La organización, la recopilación y la publicación de estadísticas reflejan las dificultades que existen para crear instituciones administrativas, las cuales requieren estabilidad y continuidad. A pesar del deseo constante de producir estadísticas nacionales estandarizadas y continuas en el siglo XIX, la creación de un servi- cio de estadistica nacional fue una tarea prolongada y ardua para los intelectuales y políticos mexicanos.

La presentación oral de información basada en bibliografía puede ser un proyecto problemático; aburrido en extremo, para ser francos. Por su naturaleza, la representación y el análisis bibliográficos se presentan mejor por escrito y visualmente a modo de que el lector interactúe con las fichas registradas. Bibliotecarios e investigadores interpretan la gama y la variedad de la información bibliográfica sin mediación, por secuencia y conforme a la escuela gestalt. 
En esta presentación, trataré de resumir la naturaleza de la folletería desde un punto de vista topográfico. Indicaré el número de registros bibliográficos de cada periodo. Con base en las divisiones cronológicas históricas, presentaré brevemente los acontecimientos institucionales que se refieren a la publicación de estadísticas durante cada periodo, y después señalaré la importancia de esta literatura. En ocasiones incluiré algún comentario sobre determinados tipos de folletos. Por la necesidad de ser concisos, en esta presentación no se hará referencia directa a la ubicación de las publicaciones o tipógrafos e impresores.

En la sección siguiente trataré algunos temas y problemas bibliográficos inherentes a la elaboración de la base de datos derivada del uso académico de los folletosy, por último, sugeriré algunas directrices para seguir elaborando en el futuro la base de datos específica para materiales relacionados con la estadística.

Debido a que ésta es sólo una presentación preliminar y se enfoca a la información bibliográfica extraída de la base de datos, omití el a parato académico de notas, citas, etc.; sin embargo, incluí una bibliografia de fuentes que han sido de gran utilidad en esta presentación y que pondrán al alcance de los interesados el tema de la historia de las publicaciones estadísticas.

PERIODOS CRONOLÓGICOS

1821-1837: 22 fichas

Durante el siglo XIX, todas las constitu- ciones mexicanas estipulaban el levantamiento de un censo cada diez años. Los censos exactos de la población estatal se consideraban vitales para la organización de congresos representativos. La administración pública después de la independencia, en especial en la república federal, no tenía la capacidad para manejar la recopilación y la publicación sistemáticas de las estadísticas nacionales. El primer paso hacia la publicación sistemática de estadísticas llegó con la creación de la Sociedad Mexicana de Geografia y Estadística en 1833, pero las publicaciones de importancia no comenzaron sino hasta la década de 1860 .

En la época de la independencia, las publicaciones fueron principalmente trabajos retrospectivos de Humboldt e ideas de la estadística de Nueva Españay del Resumen de la Estadística del Imperio Mexicano 1822 de Simón Tadeo Ortiz de Ayala. Sin embargo, sorprenden los intentos generalizados de publicar datos estadísticos vigentes durante los primeros diez años de independencia. Querétaro, Durango, 'Tabasco, Occidente, Veracruz, Oaxaca y Sonora publicaron estadísticas. El trabajo de mayor influencia en este periodo fue el de Michoacán en 1822. Durante muchos años, la mayor parte de los estudios y de las publicaciones estadisticas fueron regionales y el estado era la unidad territorial básica.

1838-1853: 25 fichas aprox.

Con el establecimiento de la república central, se consideró adecuado resta- 
blecer las formas coloniales de la presentación de estadísticas. Sin embargo, la república conservadora nunca llevó a cabo con éxito un censo demográfico del país. Por otra parte, los informes presentados por los sacerdotes de las parroquias no eran lo suficientemente completos como para cumplir con las normas estadísticas europeas.

El principio de la década de 1830 y la época posterior a la interrupción de la guerra con Estados Unidos marcaron otro ciclo de estadísticas estatales. Estos diferentes compendios, empezando con los títulos de "noticias", "ensayo", "estudio" o "apuntes", fueron publicados por personas de los estados de Oaxaca, Jalisco, San Luis Potosí, Querétaro, Tlaxcala, Durango, Sonora y Sinaloa. La uniformidad de sus títulos y su permanencia en un periodo tan prolongado hacen pensar en un origen común. ¿Cuáles fueron los antecedentes intelectuales e institucionales y qué fue lo que motivó la publicación de este tipo de obras en esos estados?

\section{4-1867: 11 fichas}

Como muestra el bajo número de fichas en este periodo, la turbulencia de las décadas intermedias de guerras extranjeras -incluidas invasiones-y de la guerra civil, destruyó el sistema colonial de presentación y elaboración de estadísticas que se había reactivado. Sin embargo, se tuvo la oportunidad de crear instituciones de recopilación y publicación de estadísticas durante un breve periodo. La creación del Mi- nisterio de Fomento en 1853 y la realización de su Memoria en 1857 dio como resultado la primera fuente importante de datos estadísticos nacionales sobre demografia, economía y sociedad que reflejaba el interés de proporcionar datos para optimizar la toma de decisiones en materia de política pública. Junto con la Memoria de Fomento, aparecieron los primeros folletos de orientación nacional de Antonio García Cubas y Miguel Lerdo de Tejada en 1856 y 1857.

\section{8-1880: 18 fichas aprox.}

A pesar de la gran reorganización política del México independiente, las técnicas de administración se aplicaron en forma gradual y sólo empezaron a reflejar la modernidad del periodo alrededor de 1870. Durante la época de la república restaurada surgieron las importantes memorias financieras y económicas del Ministerio de Hacienda de Míguel Lerdo de Tejada, Matías Romeroy Emiliano Busto. En el contexto del federalismo, el levantamiento de censos se delegó a los estados, que determinaron la forma y la exactitud de los datos. La coordinación de esfuerzos entre los estados se vio obstaculizada en la etapa inicial e intermedia de inestabilidad. En su mayor parte, las cifras demográficas plausibles en los estados y los territorios dependieron de la esporádica cooperación entre organismos públicos, intelectuales dedicados y sociedades.

Antonio García Cubas, folletista inveterado, promovió la cooperación y 
la estandarización entre los gobiernos estatales, así como su participación en la aportación de datos estadísticos. Además de los esfuerzos de García Cubas, la folletería reflejó la naturaleza altamente fragmentada y desigual entre estados y localidades. El principal acontecimiento fue el inicio de las estadísticas electorales y el análisis de las divisiones de los territorios electorales de Basilio Pérez Gallardo en la década de 1870.

\section{1-1892: 32 fichas}

Durante la administración de Manuel González, que se caracterizó por numerosos e importantes logros institucionales y de obras públicas, se creó la Dirección General de Estadística, bajo los auspicios del Ministerio de Fomento, seguida de la publicación nacional de estadísticas continuas a partir de 1884 en adelante.

Naturalmente, las leyes, los reglamentos y demás publicaciones sobre la organización y la instrumentación del nuevo servicio de estadística nacional dominaban la producción de folletos. También se publicaron estos títulos a escala regional. Un estudio acerca de qué estados publicaron leyes y reglamentos arrojará luz sobre el apoyo estatal a la nueva dependencia y sus iniciativas.

\section{3-1910: 46 fichas}

Además de varias series de estadísticas continuas, en el periodo de los gran- des censos de población nacionales de 1895, 1900 y 1910 predominó la publicación de folletos a escala nacional y regional, que incluían instrucciones, resúmenes estatales y otros análisis derivados de los censos. Asimismo, surgió la publicación de monografias de estadísticas nacionales y regionales relacionadas con la iglesia. Por último, las estadísticas sociales empezaron a proliferar y cubrian los siguientes rubros: Nacimientos y defunciones, Tasas de morbilidad, Salud y hospitales.

ELABORACIÓN DE BIBLIOGRAFÍAS

Y BASES DE DATOS

La investigación bibliográfica ha revelado que, al parecer, se han omitido algunos folletos y, en otros casos, los estudiosos citan obras que parecen ser folletos pero que no se mencionan en ninguna otra fuente. Se omitieron dos títulos de García Cubas, y Viviane Bracher de Márquez indica en su bibliografia que existe una publicación de 118 páginas de la Memoria monumental de 1880 de Emiliano Busto en la biblioteca de la Sociedad Mexicana de Geografia y Estadística. Está porverse si ésta es una copia verdadera (separata) o una parte tomada de la obra en tres volúmenes. Sólo una investigación directa puede despejar esta posibilidad intrigante. Aunque de menor importancia, éstos son problemas típicos del trabajo bibliográfico en general.

El folleto de Francisco Barrera Lavalle, que por escaso margen queda fuera de la cronología del proyecto, ha soportado la prueba del tiempo y sigue 
siendo una fuente fundamental para iniciar la investigación de la estadística mexicana. En su obra desarrolla una taxonomía de las tres clases de documentos: 1) apuntes, 2) memorias y 3) estadísticas per se. Aunque de extrema importancia como fuente de estadística, la Memoria es un tipo de publicación muy intrincada para la base de datos, el objeto de la estadística y, sin duda, para varias categorías más de la información bibliográfica.

Esto se aplica de manera especial a la obra de Brachet de Márquez, quien utiliza apuntes con regularidad, aunque la fuente principal del gobierno estatal es la Memoria. Muchos de los documentos estatales incluyen menos de 100 páginas y la mayor parte de las primeras memorias federales anteriores a la década de 1850 tienden a ser breves y cumplen cabalmente con los criterios estipulados para el folleto.

Las memorias son la laguna más evidente en la base de datos. Esta omisión se deriva de la convención adoptada por la mayor parte de las bibliotecas que consiste en tratar todas las memorias como publicaciones seriadas. En las extracciones automatizadas y manuales no se incluyeron las fichas de las memorias. Es posible extraer estas fichas a escala federal y estatal sin gran dificultad, pero deberá mediar una descripción monográfica completa.

Por último, nos planteamos la pregunta antropológica, "¿qué parece faltar?” ¿Podemos preguntar dónde están Lucas Alamán, José María Luis Mora y Manuel Payno? A pesar de largas historias de escritos oficiales y polémicos es notable su ausencia como autores de cualquiera de las publicaciones. En es- te caso, ¿pudiera ser que su aportación principal a la estadística se haya dado como autores oficiales de memorias? ¿Acaso sus escritos partidarios sólo se publicaron en periódicos y obras más grandes? En realidad, ¿por qué faltan?

\section{FUENTES Y BIBLIOTECAS FUTURAS $O$ FOLLETOS ESTADÍSTICOS}

Woodrow Borah ha clasificado Ia Bibliografia Mexicana de Estadística como una bodega virtual de material estadístico. Integrada por miles de fichas de varias de las principales bibliotecas mexicanas y extranjeras, una revisión superficial de las diferentes secciones de esa bibliografía revela que es muy probable extender la base de datos. Su principal deficiencia es que muchas de las fichas carecen de fecha, por lo que es necesario buscar en bases de datos o en catálogos de bibliotecas ya publicados.

Biblioteca de la Sociedad Mexicana de Geografia y Estadística (SMGli). Hasta la creación y la operación de la Dirección General de Estadística después de 1882, la SMGE era la mejor fuente para consultar las cifras demográficas del siglo XIX. La institución se conoce no sólo por la amplia gama de sus publicaciones, sino por el gran número de periódicos y folletos adquiridos mediante acuerdos de canje con México, así como con el exterior. Antes de la creación de la DGE, esta biblioteca funcionaba como un depósito importante de datos estadísticos sobre México.

Biblioteca del INEGI. Permítanme usar este foro para hacer un comentario editorial. El sismo de 1985 destru- 
yó el catálogo de tarjetas de esta rica colección y ha hecho prácticamente imposible el acceso de materiales históricos. Ya en 1993, utilizando referencias tomadas de antiguos catálogos publicados de la DGE, no fue posible recuperar ni siquiera las publicaciones que se encontraban en los estantes que el visitante podía ver desde la recepción. El acceso normativo al material de estas bibliotecas es esencial para cualquier investigación cuantitativa de índole histórica.

\section{CONCLUSIÓN}

EI Instituto Mora, al elaborar la base de datos, afirma tanto el papel de la folletería en la historia de las publicaciones estadísticas mexicanas, como la diversidad de la de la historia cuantitativa. La base de datos, aunque fragmentaria por el momento, ha conjuntado una serie de publicaciones similares y variadas que forman parte de distintas bibliografías y trabajos académicos. Como he señalado en forma sucinta en el texto, las extraordinarias y evidentes relaciones que existen entre varias de las publicaciones sugieren el valor académico de la investigación de este material estadístico impreso.

BIBLIOGRAFÍA SEIECTA SOBRE LA eSTADísTICA EN MÉXICO

-Barrera Lavalle, Francisco, Apuntes para la historia de la estadística en México,
1821 a 1910, Tip. de la Vda. De F. Díaz de León, sucs., México, 1911.

-Cook, Sherburne F.y Woodrow Borah, "Materials for the demographic history of Mexico, 1500-1960", en Essays in population history: Mexico and the Caribbean, Berkeley, University of California Press, Los Ángeles, 1971. vol. 1, pp. 1-72.

-Cortines, Adolfo Ruiz y Luis Híjar y Haro, "La estadística; su evolución en México", en Primer centenario de la Sociedad Mexicana de Geografía y Estadística, 1833-1933, smGe México, 1933, t. I, pp. $409-456$.

-Davis, Keith, "Tendencias demográficas urbanas durante el siglo XIX en México", Historia Mexicana, vol. Xxl, núm. 3, enero-marzo 1972, pp. 481-524.

-Flores Talavera, Rodolfo, "Historia de la estadística nacional", Boletín de la Sociedad Mexicana de Geografía y Estadística, t. LXXXVI núms. 1-3, julio-diciembre 1958 , pp. 13-45.

-"Indice general que comprende desde el tomo I hasta el LXII 1839-1947", Boletín de la Sociedad Mexicana de Geografía y Estadística, t. IXIV, núms. 1-3, juliodiciembre de 1947.

-Kicza, John, "Mexican demographic history in the nineteenth century: evidence and approaches", Statistical abstract of Latin America, 1981, vol. 21, pp. 593 609.

-Márquez, Viviane Brachet de, La población de los estados mexicanos en el siglo XIX (1824-1895), Departamento de Investigaciones Históricas-Instituto Nacional de Antropología e Historia/SEP, México, 1976.

-México, Dirección General de Estadística, Bibliografĩa mexicana de estadísti$c a$, Talleres Gráficos de la Nación, México, 1941-1942, 2 vols. 